\title{
BMJ Open High-intensity interval exercise training before abdominal aortic aneurysm repair (HIT-AAA): protocol for a randomised controlled feasibility trial
}

\author{
Garry A Tew, ${ }^{1}$ Matthew Weston, ${ }^{2}$ Elke Kothmann, ${ }^{3}$ Alan M Batterham, ${ }^{4}$ \\ Joanne Gray, ${ }^{5}$ Karen Kerr, ${ }^{6}$ Denis Martin, ${ }^{4}$ Shah Nawaz, ${ }^{7}$ David Yates, ${ }^{8}$ \\ Gerard Danjoux ${ }^{3}$
}

To cite: Tew GA, Weston M, Kothmann $\mathrm{E}$, et al. Highintensity interval exercise training before abdominal aortic aneurysm repair (HITAAA): protocol for a randomised controlled feasibility trial. BMJ Open 2014;4:e004094. doi:10.1136/ bmjopen-2013-004094

- Prepublication history and additional material for this paper is available online. To view these files please visit the journal online (http://dx.doi.org/10.1136/ bmjopen-2013-004094).

Received 25 September 2013 Revised 7 December 2013 Accepted 11 December 2013

\section{CrossMark}

For numbered affiliations see end of article.

Correspondence to Dr Gerard Danjoux; Gerard.danjoux@stees.nhs.uk

\section{ABSTRACT}

Introduction: In patients with large abdominal aortic aneurysm (AAA), open surgical or endovascular aneurysm repair procedures are often used to minimise the risk of aneurysm-related rupture and death; however, aneurysm repair itself carries a high risk. Low cardiopulmonary fitness is associated with an increased risk of early post-operative complications and death following elective AAA repair. Therefore, fitness should be enhanced before aneurysm repair. High-intensity interval exercise training (HIT) is a potent, time-efficient strategy for enhancing cardiopulmonary fitness. Here, we describe a feasibility study for a definitive trial of a pre-operative HIT intervention to improve post-operative outcomes in patients undergoing elective AAA repair.

Methods and analysis: A minimum of 50 patients awaiting elective repair of a $5.5-7.0 \mathrm{~cm}$ infrarenal AAA will be allocated by minimisation to HIT or usual care control in a 1:1 ratio. The patients allocated to HIT will complete three hospital-based exercise sessions per week, for 4 weeks. Each session will include 2 or 4 min of high-intensity stationary cycling followed by the same duration of easy cycling or passive recovery, repeated until a total of 16 min of high-intensity exercise is accumulated. Outcomes to be assessed before randomisation and 24-48 $\mathrm{h}$ before aneurysm repair include cardiopulmonary fitness, maximum AAA diameter and health-related quality of life. In the postoperative period, we will record destination (ward or critical care unit), organ-specific morbidity, mortality and the durations of critical care and hospital stay. Twelve weeks after the discharge, participants will be interviewed to reassess quality of life and determine post-discharge healthcare utilisation. The costs associated with the exercise intervention and healthcare utilisation will be calculated.

Ethics and dissemination: Ethics approval was secured through Sunderland Research Ethics Committee. The findings of the trial will be disseminated through peer-reviewed journals, and national and international presentations.

Trial registration: Current Controlled Trials ISRCTN09433624.

\section{BACKGROUND}

Major non-cardiac surgery is associated with a substantial peri-operative risk; the overall mortality rate appears low $(c .1-2 \%)$, but the number of operations performed (c. 250 million per annum worldwide) results in a large absolute number of deaths. ${ }^{1}$ Moreover, post-operative complications occur up to five times as frequently, ${ }^{1}$ with survivors experiencing physical limitations and reduced life expectancy. ${ }^{23}$ Identification of individuals in this 'at-risk' group for death and complications creates a significant challenge to clinicians in the pre-operative period. Objective assessment of cardiopulmonary fitness in the pre-operative period utilising cardiopulmonary exercise testing (CPET) is the established gold standard across the UK. It has a developing evidence base in predicting adverse outcome across a variety of high-risk surgical procedures, ${ }^{4}$ and this has contributed substantially to clinicians' understanding of the impact of poor cardiopulmonary fitness.

There is a convincing physiological rationale linking improved cardiopulmonary fitness to a reduction in adverse outcome following surgery. The surgical stress response involves neuroendocrine, metabolic and inflammatory effects leading to a catabolic state and increased basal metabolic rate (up to three times pre-operative values ${ }^{5}{ }^{6}$ ). A patient with adequate cardiopulmonary fitness is able to meet these extra demands post-operatively, but patients with inadequate fitness levels might be unable to cope, leading to tissue hypoxia and peri-operative complications. Approximately, half of the patients presenting for intra-abdominal surgery do not have the prerequisite fitness, on objective exercise testing, to be deemed 'low risk' for peri-operative complications. ${ }^{7}$ It 
is intuitive that improving fitness levels in the pre-operative period will translate to reduced death and complications following major surgery.

Little is known about the impact of pre-operative exercise training on post-operative outcomes. In a recent systematic review, ${ }^{8}$ the authors concluded that preoperative exercise therapy prior to cardiac or abdominal surgery results in a reduced hospital length of stay and reduced post-operative morbidity, but that more research is required on the impact and long-term benefits. However, of the five studies identified that focused on cardiac and abdominal surgery, four involved inspiratory muscle training with pulmonary complications as the primary outcome. Clearly, this intervention improves respiratory muscle function, which might reduce postoperative pulmonary morbidity. However, this form of training is unlikely to favourably influence the wider range of sequelae of the surgical stress response. Only one study focused on the effect of a more general presurgery exercise training intervention. Arthur et at reported a reduction in median hospital length of stay of 1 day (vs usual care control) following coronary artery bypass graft surgery with an 8-10 week exercise training programme plus education reinforcement and social support. The intervention involved $30 \mathrm{~min}$ of aerobic interval training, performed twice a week at $40-70 \%$ of functional capacity.

We believe that a programme of research is needed now to evaluate the benefits of pre-operative exercise training in patients undergoing elective noncardiothoracic surgery. Abdominal aortic aneurysm (AAA) is a frequently lethal disease occurring in $~ 5 \%$ of men aged 50-79 years. ${ }^{10}$ Annually, 5000-6000 surgical repairs are performed across the UK, ${ }^{11}$ making this an ideal homogenous high-risk target population. The incidence of comorbid disease is higher than other agematched surgical populations: cardiac disease $60-70 \%$, respiratory disease $40-50 \%$, long-term smoking $50-80 \%$, renal disease $10-12 \%$ and diabetes $10-12 \% .{ }^{12}$ Anecdotal observation from $>1000$ CPETs confirms that this population is also substantially less fit than other age-matched surgical populations. Findings from two recent publications also support the adverse impact of poor fitness on outcome in patients undergoing AAA repair. ${ }^{13} 14$

Intervention for AAA can be performed by either open or endovascular repair (EVAR), with a current ratio nationally of 55:45 in favour of EVAR. ${ }^{11}$ Thirty day mortality for open surgery in the UK in 2008 was $7-8 \% .^{12}$ Earlier studies from the $\mathrm{USA}^{15}$ and the Netherlands ${ }^{16}$ reported major post-operative morbidity of $30-40 \%$. Endovascular treatment is less invasive, with mortality and cardiorespiratory morbidity rates of $2-3 \%^{11}{ }^{12}$ and $10-15 \%,{ }^{15}{ }^{16}$ respectively. For open surgery, the UK mortality rate was higher than expected with respect to comparable countries, prompting the publication of a quality improvement programme document with the explicit remit of standardising management to improve outcome. ${ }^{11}$ Encouragingly such standardisation has brought about a significant 30-day mortality benefit for both procedures in the Vascular Society's most recent publication: $4.3 \%$ and $0.9 \%$ for open AAA and EVAR, respectively. ${ }^{17}$ Despite this, there remains significant room for improvement. No information is routinely available on non-fatal complications, which are up to five times more prevalent than mortality and known to affect patient's quality of life and overall life expectancy on hospital discharge. In addition, a key omission from the guidance is evidence or advice in relation to improving pre-operative fitness, despite the fact that one of the main conclusions of the EVAR-2 study was that vascular teams should be focusing on techniques to improve patient's fitness pre-operatively. ${ }^{18}$

The proposed benefits of exercise 'prehabilitation' are mediated by increases in cardiopulmonary fitness. Two pilot randomised controlled trials (RCTs) undertaken by our research team have documented improvements in cardiopulmonary fitness following moderate-intensity endurance exercise training in patients under surveillance for a small AAA. Kothmann et $a l^{19}$ reported a $10 \%$ increase in the oxygen consumption at the ventilatory threshold (a submaximal marker of cardiopulmonary fitness of prognostic significance) after 6 weeks of moderate-intensity cycling exercise performed for $30 \mathrm{~min}$ twice weekly. Tew et $a l^{20}$ observed a $2.5 \mathrm{~mL} / \mathrm{kg} / \mathrm{min}$ $(\sim 20 \%)$ improvement in the ventilatory threshold after 12 weeks of moderate-intensity cycling and treadmill walking exercise performed for 35-45 min thrice weekly. A recent review of pre-operative exercise training ${ }^{21}$ proposed a research agenda, with future directions including the role of prehabilitation in improving fitness levels prior to major surgery, the use of robust study designs with appropriate outcome measures and evaluations of the effects of high-intensity interval exercise training (HIT) as a model for which there is extensive evidence of benefit in other patient groups, including patients with heart failure. ${ }^{22}$ A recent literature review by Guiraud et $a l^{23}$ in cardiac rehabilitation concluded that when compared with moderate-intensity training, HIT has a similar safety profile (low absolute risk) and produces greater and more time-efficient improvements in fitness. For the current proposal, HIT therefore represents a particularly attractive approach, as the time-window for intervention once a patient has been identified for aneurysm repair might be as short as 4-6 weeks. ${ }^{11}$ Therefore, an intervention with the potential for more rapid fitness benefits is preferable.

Our programme of work is aligned to the Medical Research Council's (MRC) guidance for developing and evaluating complex interventions. ${ }^{24}$ Given the limited extent of the evidence base, a feasibility study is clearly required to inform a subsequent definitive trial. The MRC guidance stresses that crucial feasibility work is often absent or insufficient, with 'definitive' trials undermined by acceptability, adherence, and delivery of the intervention, recruitment and retention issues and smaller than expected effect sizes. 
We are conducting a feasibility study to explore the potential benefits of a 4-week HIT programme, delivered prior to surgery for AAA repair. This will be stationary, cycle-based, in-hospital and undertaken three times/week.

\section{AIMS}

\section{Explore potential primary outcomes for a subsequent definitive RCT}

The physiological rationale suggests a causal pathway between adaptations consequent to exercise training and reduced mortality and morbidity. The potential primary outcomes for a definitive trial therefore include 30-day mortality, morbidity (Post-Operative Morbidity Survey (POMS) score), health-related quality of life (HRQOL), hospital length of stay, costs and cost-effectiveness.

\section{Examine the suitability of the exercise training for a subsequent definitive RCT}

HIT shows much promise as an efficacious, time-efficient and also enjoyable intervention for improving fitness. However, it has not been employed with patients with AAA awaiting repair.

\section{Examine the willingness of patients to be randomised and explore potential patient preferences}

In RCTs, the patients might have strong treatment preferences resulting in a refusal to be randomised, affecting the generalisability of the results. Or, they might agree to be randomised but suffer from 'resentful demoralisation' if they end up in the non-preferred arm of the trial, leading to poor adherence. This issue requires examination in a feasibility study, as the preference effects for exercise versus control in this patient population are unknown. Theoretically, the patients might have a preference for the exercise arm due to a belief in the benefits. Notwithstanding the patient's information provided, others might be fearful of engaging in high-intensity exercise prior to surgery and therefore might exhibit a preference for the control arm. These issues could affect the success of a definitive trial.

\section{OBJECTIVES}

\section{Aim 1}

To define the characteristics of the potential outcome measures. Specifically,

1. Define the distribution (eg, log-normal, Poisson, etc for, eg, length of hospital stay) and estimate the variability for the potential primary outcome measures to inform sample size planning for a subsequent definitive trial.

2. Estimate the effect size (intervention minus control) for each potential outcome variable (point estimate and its uncertainty). This information reflects the effectiveness of the intervention and the 'noise' in the measurement (precision of the measure) and together with the other information will inform the choice of primary outcome for a subsequent trial.
3. Assess the ease of data collection for each potential primary outcome (including participant and clinician burden, assessed via qualitative data).

\section{Aim 2}

This will specifically include objective fitness changes, safety, enjoyment, delivery and adherence of the exercise intervention.

\section{Aim 3}

To examine the strength of patient's preferences for either the intervention or control arms (qualitative data). These data will be elicited from all patients assessed as eligible, to determine the extent to which preferences affect recruitment and adherence.

\section{METHODS/DESIGN \\ Study design}

Three-centre, two-arm, parallel-group, randomised, controlled feasibility study. The study flowchart is shown in figure 1 .

\section{Study population and recruitment routes}

Patients being assessed for surgery for a $5.5-7.0 \mathrm{~cm}$ AAA will be approached via vascular surgical or preoperative assessment clinics at recruiting institutions. The potential recruits will be approached at this stage by a study investigator and, if interested, provided a study information sheet. Where an investigator is not available, a study information letter will be sent to the patient requesting permission to contact them about the study.

\section{Sample size}

The sample size for a feasibility study should be adequate to estimate critical parameters with sufficient precision. Herein, the critical outcome is adherence to the exercise intervention. A patient will be deemed compliant if they complete $\geq 75 \%$ of the scheduled sessions, that is, $9 / 12$ sessions for a 4-week intervention, plus all once-weekly maintenance sessions if surgery is delayed. We define success with respect to adherence as a lower limit of $0.67(c .2 / 3$ of the population) for the $90 \%$ CI for the proportion of the exercise group complying with the intervention. We estimate that $\geq 85 \%$ of the exercise group will be 'compliers' based on our pilot studies in patients with small AAA. ${ }^{19} 20 \mathrm{~A}$ $90 \%$ CI for a single proportion around a value of 0.85 is $0.68-0.95$, with $\mathrm{n}=25$ patients. Using a $1: 1$ allocation ratio, we require 25 patients/trial arm, 50 in total.

A minimum of 50 participants will therefore be recruited to the study.

Approximately 150 repairs of AAA $<7 \mathrm{~cm}$ are performed across our three clinical institutions annually. Assuming 30\% attrition for specialist referral and lack of prerequisite fitness, approximately 176 potential patients will be available in our 21-month recruitment time-window. 
Figure 1 Flowchart of the study. AAA, abdominal aortic aneurysm; EVAR, endovascular aneurysm repair; POMS, post-operative morbidity survey.

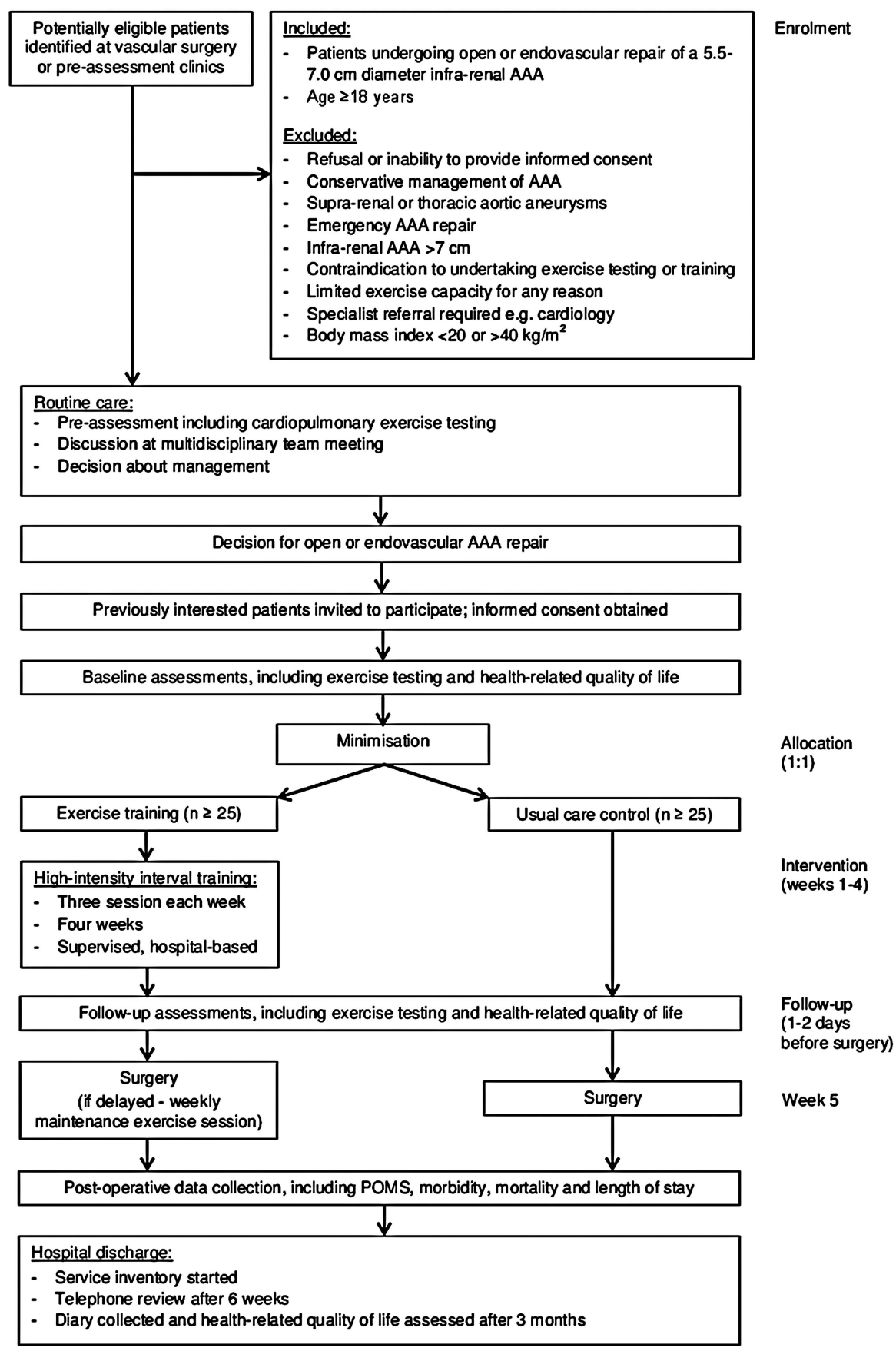

\section{Clinical assessment}

The clinical assessment processes described are based on routine practice at the recruiting institutions. All patients who are being considered for an elective AAA repair will attend a pre-operative assessment clinic. Here, an individual's clinical risk profile for surgery will be established using a history of relevant comorbidities, physical examination and CPET. Evidence-based optimisation of medication will be performed at this stage.

After risk profiling, a patient's treatment options are discussed in a vascular multidisciplinary team meeting comprising input from surgery, anaesthesia and radiology. A risk-benefit assessment is undertaken based on a nationally agreed care pathway. ${ }^{11}$ There are three possible outcomes: open aneurysm repair, EVAR or conservative management (ie, when surgical risk is deemed too great or the patient elects not to proceed). The most appropriate post-operative care facility is also determined.

\section{Eligibility criteria}

Recruitment

Patients who express an interest at the clinic, or who are sent a study information letter, will be contacted by telephone inviting participation (if no exclusion criteria). With verbal consent, the baseline assessment will be scheduled (see figure 1). 


\section{Baseline assessment}

Written informed consent will be obtained. The participants will confirm their medical history and the current medication and undergo a physical examination. Baseline measurements will then be recorded, including

- The patient characteristics (sex, stature, body mass and body mass index).

- Resting pulse, blood pressure and oxygen saturations.

- Maximum AAA diameter via transabdominal ultrasound (not if an ultrasound scan has been performed within the previous 8 weeks).

- Cardiopulmonary fitness via CPET. The CPET data will be used to identify the intensity at which the patients in the exercise group will initiate training. We have previously demonstrated the reliability of CPET in patients with AAA. ${ }^{25}$ Testing will be performed according to an agreed protocol across all recruiting sites (available on request).

- HRQOL using the Medical Outcomes Study SF-36 V.2 questionnaire and the EuroQol EQ-5D-5L questionnaire, both of which have been used previously in patients with AAA. ${ }^{18} 2627$

- Participant's preference for 'exercise' training or 'usual care'-prior to randomisation to explore patient's preferences and subsequent changes in attitude consequent to the intervention. This design permits the exploration of the effects of preference in the analysis. ${ }^{28}$

\section{Randomisation}

After baseline assessment, patients will be randomly allocated 1:1 to exercise or usual care control (no supervised exercise), using minimisation to ensure balance across trial arms for important prognostic factors. We do not list these factors here, to avoid any risk of the staff recruiting patients being able to decipher the allocation sequence. Full details of the minimisation process will be published in a separate document with restricted access. The study statistician (AMB) will conduct the minimisation process remotely via email.

\section{Exercise intervention (weeks 1-4)}

The exercise intervention period will be for 4 weeks leading up to surgery. Where possible, the participants (exercise and control) will have a surgical date booked for the following week (week 5).

The exercise programme is broadly based on that which has been shown to be safe and effective for improving cardiopulmonary fitness in patients undergoing cardiac rehabilitation. ${ }^{22} 29-31$ The patients allocated to the exercise group will complete three sessions of hospital-based HIT per week, throughout the 4-week pre-operative period. Exercise will be stationary cycling (Optibike Med, Ergoline, Germany), which has been reported to be a preferred mode for vascular patients (unpublished observations). Each session will begin and end with 10 and 5 min of unloaded cycling, respectively. In the first week of training, the main body of each session will involve eight 2 min bouts of cycling, interspersed with 2 min periods of unloaded cycling or 'off-the-bike' slow walking, depending on the patient's preference. All of the 'work' bouts during the first session will be performed at the power output associated with ventilatory threshold determined on baseline CPET (ie, the demarcation between moderate and heavy exercise intensity domains ${ }^{32}$ ). In subsequent sessions, power output will be gradually manipulated until the patient reports a perceived exertion of 6-7 on Borg's CR-10 scale $^{33}$ (ie, hard to very hard) at the end of each work interval. However, for safety reasons, the intensity of exercise will be made easier if systolic blood pressure exceeds $180 \mathrm{~mm} \mathrm{Hg}^{34}$ or if heart rate exceeds $95 \%$ of the maximum observed on baseline CPET. In weeks 2-4, and for variety, the patient will be allowed to choose between doing four 4 min work bouts or eight 2 min bouts as the main body of each exercise session, both with a 1:1 work-to-rest ratio. Thus, each session will last $\sim 45$ min regardless of patient's choice, which will include $16 \mathrm{~min}$ of high-intensity exercise. An experienced physiotherapist will supervise each session and record power output, perceived exertion and blood pressure (manual sphygmomanometer) at the end of each work interval. Heart rate will be recorded continuously at $5 \mathrm{~s}$ intervals through the entire exercise session (Polar RS400, Kempele, Finland). The collection of such data will permit a detailed quantification of the exercise intervention. The patients who do not undergo surgery in week 5 will complete one HIT session per week up until surgery to maintain fitness. ${ }^{35}$ All adverse events will be recorded.

Information in relation to participant's perceived enjoyment of exercise is important to monitor. This is of relevance in adherence to the programme, while providing valuable information for planning a definitive study. We therefore plan to assess:

1. Changes in enjoyment of exercise within sessions and throughout the programme. Perceived or likely enjoyment can change prior, during and after exercise as a consequence of a variety of factors, for example, anxiety, enjoyment and fatigue. We therefore plan to ask participants to assess enjoyment/perceived enjoyment of exercise prior to starting, during and $20 \mathrm{~min}$ after exercise using a simple validated rating scale. ${ }^{36}$ This will take $<30 \mathrm{~s}$ to administer on each occasion and will be performed during the first (baseline), $3 \mathrm{rd}, 6 \mathrm{th}, 9$ th and 12 th sessions to track changes consequent to the programme.

2. Overall enjoyment of the exercise programme will be assessed during the week 5 assessment, using the validated multidimensional Physical Activity Enjoyment Scale (PACES). ${ }^{37}$ This will be $2-3$ days following completion of the overall programme, allowing participants' reflection and evaluation time (see week 5 assessment below).

Two experienced exercise scientists will be responsible for ensuring treatment fidelity of the exercise programme (GAT, MW). 


\section{Safety considerations}

There are two main safety considerations regarding running a programme of HIT in patients with AAA disease:

1. Cardiac risk-as previously outlined, $60-70 \%$ of individuals with AAA disease suffer from comorbid cardiac disease. The baseline assessment of clinical risk profile and CPET will be instrumental in assessing this risk in detail, under the stress of exercise, at the outset. A recent review by Guiraud $e t a t^{23}$ highlights the safety of HIT in patients with underlying coronary disease, heart failure and patients at risk of cardiovascular disease. We are reassured by their conclusion which states that "there is growing scientific evidence that HIT presents little danger for selected stable cardiac patients, provided that the prescribed protocols are respected."

In the event of an adverse cardiac event, we will have the following in place:

- Staff experienced and Intermediate Life Support-trained;

- Resuscitation equipment and oxygen immediately available;

- Hospital cardiac arrest team fully informed of time and place of training sessions;

- Participants requiring further assessment will be transferred to the accident and emergency unit within the relevant hospital.

All institutions have cardiology expertise immediately available on site.

2. Risk of AAA expansion or rupture-an intuitive concern regarding exercise testing and training in patients with aneurysms is of excessive rises in the double-product (systolic blood pressure xheart rate) evoking aneurysm expansion and rupture. However, the available evidence suggests that these concerns are unfounded. For example, no aneurysm ruptures or excessive aneurysm growth rates were reported in any of the three studies of moderate-intensity exercise training in patients with early AAA disease (from $>4000$ exercise sessions ${ }^{19} 2038$ ), two of which were conducted at our institutions. ${ }^{19}{ }^{20}$ Further unpublished data from our institutions indicate no ruptures from $>1300$ maximal exercise tests in patients with large asymptomatic AAA being considered for elective aneurysm repair. Moreover, through national networked connections, and other relevant publications, we believe this position of safety to be correct for $>5000$ maximal exercise tests. This position is also supported by the European guidelines for the management of AAA disease, which state that physical activity is not associated with AAA growth, ${ }^{39}$ as well as the American College of Cardiology/American Heart Association Practice Guidelines for the Management of Patients with Peripheral Vascular Disease,${ }^{40}$ which suggest that patients with AAA should not be fearful of vigorous activity. The only reported adverse event we are aware of came from the patients series of Best et $a t^{11}$ where a rupture occurred $12 \mathrm{~h}$ after maximal exercise testing in a patient with a $6.1 \mathrm{~cm}$ AAA, yielding a rupture rate for this study of $0.4 \%$. However, the causal relationship between exercise and rupture is difficult to establish given the $12 \mathrm{~h}$ intervening period.

It would therefore appear that the risk of AAA growth or rupture is very low in the context of exercise training for individuals with small and large aneurysmal disease. This risk would appear to be $<1: 5000$. When balanced against national peri-operative mortality/morbidity figures available for surgery in the UK at present, we feel that this represents clear benefit in favour of undertaking the pre-operative exercise as set out.

In the event of a suspected AAA rupture, patients would immediately be transferred to the accident and emergency department for prompt further evaluation. All clinical institutions have on-site vascular teams able to immediately respond to such a situation.

Despite this, we see risk minimisation in this context as our primary concern and will implement the following control measures:

- Thorough preparticipation screening;

- Exclusion of high-risk patients;

- Hospital-based exercise testing and training;

- Exercise sessions supervised by experienced cardiac physiotherapists trained in Intermediate Life Support;

- Exercise sessions performed $>3 \mathrm{~h}$ after waking given the higher frequency of cardiovascular events during the morning hours ${ }^{42}$;

- Prompt evaluation of prodromal symptoms;

- Resuscitation equipment and oxygen immediately available;

- Exercise termination if a patient has signs/symptoms suggestive of distress, cardiac or AAA adverse event;

- Reduction in exercise intensity if a patient has systolic blood pressure rise to $>180 \mathrm{~mm} \mathrm{Hg}$, or heart rate $>95 \%$ of their maximum (from baseline CPET).

\section{Safety governance}

In line with MRC guidance, we have developed the following safety governance structure for the study:

- Data Monitoring and Ethics Committee (DMEC) the DMEC comprises three academics/clinicians with appropriate expertise who are independent from the running of the study. Any serious adverse events will be reported to and fully investigated by the DMEC. The DMEC will make recommendations to the Trial Steering Committee (TSC) with regard to any ethical or safety concerns they may have.

- TSC-this committee is led by the Chief Investigator (CI), with representation from all collaborating clinical and academic institutions. A lay representative will be approached to sit on the committee. The TSC will meet three times per year to discuss all elements of study progress and conduct.

- TSC Safety Committee-a formal study safety committee has been established and comprises three study 
investigators, including the CI. This group has the remit of ensuring that all appropriate safety standards are in place prior to study start and during the course of the research.

All adverse and serious adverse events will be managed within the strict governance arrangements of the study sponsor and participating clinical institutions.

\section{Week 5 assessments}

During week 5, it is anticipated that participants will undergo AAA repair. In the same week, but 1-2 days prior to surgery, assessments will be performed on all participants. The outcomes, which are primarily aimed at investigating the safety and effectiveness of the exercise intervention compared with usual care, will include

- Cardiopulmonary fitness via CPET (to assess fitness changes from baseline);

- Maximum AAA diameter via transabdominal ultrasound (to establish safety of exercise in relation to aneurysm growth);

- HRQOL questionnaires;

- PACES assessment for overall exercise programme enjoyment (exercise intervention group only).

Owing to logistical reasons, it is anticipated that not all participants will undergo surgery in week 5 . This will mainly be due to a temporary lack of hospital beds for admission pre-operatively, or critical care post-operatively. In this situation, surgery will be rescheduled, where possible, within 4 weeks. A repeat CPET will be undertaken in patients whose surgery is delayed $>4$ weeks, to ensure we have an accurate assessment of pre-operative fitness in all patients. A CPET assessment of those patients who have performed one high-intensity training session per week from week 5 onwards will provide further valuable information of the dose-response nature of high-intensity training in this population.

An overview of data to be collected for this aspect of the study can be seen on the study case report form (see supplementary file).

\section{Peri-operative period}

The participants will undergo open or endovascular aneurysm repair as determined at the multidisciplinary team meeting. All peri-operative care will be at the discretion of the vascular teams (as per usual practice) who will be blinded to group allocation. Peri-operative data will be collected as seen in Additional file 1 .

Post-operatively, an investigator blinded to group allocation will determine the following:

- Destination-ward or critical care unit;

- Post-operative Morbidity Survey Score (POMS) - a validated tool used to assess organ-specific morbidity in the post-operative period. ${ }^{43}{ }^{44}$ Scores will be collected daily;

- Mortality;

- Length of critical care and hospital stay.
Post-discharge follow-up

After hospital discharge, patients will be asked to keep a service receipt inventory to record treatment/care requirements for a 12-week period to inform the resource utilisation analysis. The participants will be interviewed via telephone at 6 weeks, with a follow-up appointment at 12 weeks after discharge to assess:

- HRQOL;

- Resource utilisation;

- Hospital readmissions, with diagnosis, within 12 weeks of initial discharge.

\section{Analysis plan of quantitative study}

Adherence with the exercise intervention will be analysed as described in the sample size justification, above, via the CI for a single proportion. For the potential primary outcomes of a subsequent definitive trial, the likely effect of the exercise intervention will be estimated via standard intervention minus control comparisons (accounting for the type of a variable and its distributional properties). We will examine the disposition of the $90 \%$ CI for the observed effect to the minimum clinically important difference for each variable. Inasmuch as this is a feasibility study, these comparisons are exploratory and are intended to inform the subsequent trial. Data management will be performed according to the governance arrangements of the institutions involved in the project.

\section{Analysis of health economics and cost-effectiveness}

A prospective economic evaluation will be rehearsed to develop and refine methods for a subsequent definitive trial. The main focus will be on how to accurately identify, quantify and value the additional costs of delivering the intervention and the potential resource implications versus usual care post-operatively and post-discharge. The costing approach will incorporate a broad analytical perspective (National Health Service and Social Services), which will help to detect cost-shifting between sectors. Resources utilised in the exercise group will be identified in terms of capital equipment and staff time. A staff-reported health economics questionnaire has been developed to facilitate this (available on request). Post-operative resource use will also be explored for all participants in terms of bed days (including critical care bed days). Post-discharge resource use for all treatment/ care related to AAA surgery will be assessed retrospectively for 12 weeks by piloting the use of a self-report Service Receipt Inventory (patient diary). This will facilitate the development of a reliable and valid tool to capture resource use. Appropriate unit costs to be applied to resource use will be identified. These will be sourced from a combination of local costings and national databases. ${ }^{45}{ }^{46}$ All costs will be combined to rehearse the methods for total health and social care cost-estimation in a subsequent definitive trial.

The methods to estimate an incremental costeffectiveness ratio for exercise versus usual care in terms 
of Quality Adjusted Life Years will be rehearsed (using EQ-5D 5L administered at baseline and 12 weeks postdischarge). In particular, issues relevant to sensitivity analysis will be explored to help understand how best to deal with statistical imprecision and other uncertainties in the full trial. For example, data will be bootstrapped to account for the expected skewness evident in economic cost data. The data collected as part of this feasibility study could be used to inform subsequent pre-trial modelling.

\section{Analysis of participant interviews}

We aim to explore the participants' post-discharge experience. From a critical realism perspective, semistructured interviews will cover quality of life, function and attitudes to exercise, including fear.

Sixteen participants will be recruited using purposive sampling and the following criteria: men and women; a range of ages; both study groups; both forms of aneurysm repair and a range of experiences post-discharge. The interviews ( $1 \mathrm{~h}$ with a research nurse; audiorecorded and transcribed) will take place in the hospital at the 12-week post-discharge visit. Transcripts will be analysed thematically using quantitative and diary data for triangulation.

Qualitative data (brief interview) will also be collected from all the participants (and from clinical outcome assessors where relevant) to assess the ease of data collection for each potential primary outcome (participant and clinician burden), to evaluate the perceived enjoyment of the exercise intervention, and to examine the strength of patient's preferences for either the intervention or control arms. These data will be elicited from all patients assessed as eligible, to help determine the extent to which preferences affect the recruitment and adherence.

\section{Criteria for success}

A subsequent definitive RCT will be deemed feasible if:

1. An appropriate primary outcome variable is defined;

2. The lower limit of the $90 \%$ CI for the proportion of the exercise intervention group complying with the intervention is $\geq 67 \%$. A patient is defined as having complied with the intervention if he completes $\geq 75 \%$ of the scheduled sessions;

3. The patient's preferences are not so strong that they result in the conclusion that an RCT is not a feasible design.

\section{Dissemination strategy}

The dissemination strategy for this research will be to inform a wide range of local, national and international audiences about the results and conclusions. It must, however, be remembered as part of this strategy that the current proposal is for preliminary work aimed at defining a subsequent definitive clinical trial.

1. Health professionals - we aim to publish our research in journals that cover the relevant medical specialties and with preference for those that deposit publications in open access databases to increase free dissemination. In addition, we aim to present this research at appropriate national and international conferences.

2. Users-from this perspective, we aim in the first instance to collaborate with our patient's representatives (from advisory and steering groups) and local experts in the patient and public involvement to best facilitate user dissemination. We plan to write a specific news piece that will be forwarded to appropriate groups and organisations.

3. Service managers-as an exploratory study, it is unlikely that results from this study will directly influence commissioning processes in the short term. Moreover, we will engage with appropriate primary and secondary care groups to discuss support for our proposed definitive study leading on from this research.

\section{Author affiliations}

${ }^{1}$ York Trials Unit, Department of Health Sciences, University of York, York, UK ${ }^{2}$ School of Social Sciences \& Law, Teesside University, Middlesbrough, UK ${ }^{3}$ Department of Academic Anaesthesia, James Cook University Hospital, Middlesbrough, UK

${ }^{4}$ Health and Social Care Institute, Teesside University, Middlesbrough, UK ${ }^{5}$ Faculty of Health \& Life Sciences, Northumbria University, Newcastle-Upon-Tyne, UK

${ }^{6}$ Department of Anaesthesia, Northern General Hospital, Sheffield Teaching Hospitals Foundation Trust, Sheffield, UK

${ }^{7}$ Sheffield Vascular Institute, Northern General Hospital, Sheffield, UK

${ }^{8}$ Department of Anaesthesia, York Hospital, York, UK

Acknowledgements The following individuals are acknowledged for making a substantial contribution to the development of this study: Louise Cawthorn, Susan Ward and Esther Carr.

Contributors GAT drafted the manuscript and contributed to the design of the study. Original study concept was by GD and AMB. All co-authors contributed to the design of the study. GD is the Chief Investigator for the project. EK is the Principal Investigator (PI) at James Cook University Hospital (South Tees), SN is the PI at Northern General Hospital (Sheffield) and DY is the PI at York Hospital. All authors contributed to the critical revision of the manuscript for important intellectual content and read and approved the final version of the manuscript.

Funding This study is funded by the National Institute for Health Research (NIHR) under its Research for Patient Benefit (RfPB) Programme (Grant Reference Number PB-PG-1111-26068). The views expressed are those of the authors and not necessarily those of the NHS, the NIHR or the Department of Health. UK National Institute of Health Research-Research for Patient Benefit Programme (PB-PG-1111-26068). The primary trial sponsor is South Tees NHS Foundation Trust (Marton Road, Middlesbrough, TS4 3BW).

Competing interests None.

Provenance and peer review Not commissioned; externally peer reviewed.

Ethics approval Ethics approval was secured through Sunderland Research Ethics Committee (reference 13/NE/0116) in May 2013.

Data sharing statement The current unpublished data for this study are available to the co-authors of this manuscript. However, data collection has not yet been completed.

Open Access This is an Open Access article distributed in accordance with the Creative Commons Attribution Non Commercial (CC BY-NC 3.0) license, 
which permits others to distribute, remix, adapt, build upon this work noncommercially, and license their derivative works on different terms, provided the original work is properly cited and the use is non-commercial. See: http:// creativecommons.org/licenses/by-nc/3.0/

\section{REFERENCES}

1. Pearse RM, Holt PJ, Grocott MP Managing perioperative risk in patients undergoing elective non-cardiac surgery. BMJ 2011;343: d5759.

2. Head J, Ferrie JE, Alexanderson K, et al. Diagnosis-specific sickness absence as a predictor of mortality: the Whitehall II prospective cohort study. BMJ 2008;337:a1469.

3. Khuri SF, Henderson WG, DePalma RG, et al. Participants in the VA National Surgical Quality Improvement Program: determinants of long-term survival after major surgery and the adverse effect of postoperative complications. Ann Surg 2005;242:326-41.

4. Hennis PJ, Meale PM, Grocott MP. Cardiopulmonary exercise testing for the evaluation of perioperative risk in non-cardiopulmonary surgery. Postgrad Med J 2011;87:550-7.

5. Viale JP, Annat GJ, Ravat FM, et al. Oxygen uptake and mixed venous oxygen saturation during aortic surgery and the first three postoperative hours. Anesth Analg 1991;73:530-5.

6. Older $\mathrm{P}, \mathrm{Smith} \mathrm{R}$. Experience with the preoperative invasive measurement of haemodynamic, respiratory and renal function in 100 elderly patients scheduled for major abdominal surgery. Anaesth Intensive Care 1988;16:389-95.

7. Wilson RJ, Davies S, Yates D, et al. Impaired functional capacity is associated with all-cause mortality after major elective intra-abdominal surgery. Br J Anaesth 2010;105:297-303.

8. Valkenet K, van de Port IG, Dronkers JJ, et al. The effects of preoperative exercise therapy on postoperative outcome: a systematic review. Clin Rehabil 2011;25:99-111.

9. Arthur HM, Daniels C, McKelvie R, et al. Effect of a preoperative intervention on preoperative and postoperative outcomes in low-risk patients awaiting elective coronary artery bypass graft surgery. A randomized, controlled trial. Ann Intern Med 2000;133:253-62.

10. Lederle F, Johnson G, Wilson S, et al. Abdominal aortic aneurysm in women. J Vasc Surg 2001;34:122-6.

11. National Abdominal Aortic Aneurysm Quality Improvement Programme Interim report. http://www.aaaqip.com/files/ aaaqip-interim-report-new-final-printed-version-23-06-11.pdf

12. Second Vascular Surgery Database Report 2008. http://www.esvs. org/sites/default/files/file/Vascunet/Vascunet\%20report\%202008.pdf

13. Hartley RA, Pichel AC, Grant SW, et al. Preoperative cardiopulmonary exercise testing and risk of early mortality following abdominal aortic aneurysm repair. Br J Surg 2012;99:1539-46.

14. Prentis JM, Trenell MI, Jones DJ, et al. Submaximal exercise testing predicts perioperative hospitalization after aortic aneurysm repair. $J$ Vasc Surg 2012;56:1564-70

15. Elkouri S, Gloviczki P, McKusick MA, et al. Perioperative complications and early outcome after endovascular and open surgical repair of abdominal aortic aneurysms. J Vasc Surg 2004;39:497-505.

16. Prinssen M, Verhoeven EL, Buth J, et al. Dutch Randomized Endovascular Aneurysm Management (DREAM)Trial Group: a randomized trial comparing conventional and endovascular repair of abdominal aortic aneurysms. N Engl J Med 2004;351:1607-18.

17. Outcomes after Elective Repair of Infra-renal Abdominal Aortic Aneurysm. http://www.vascularsociety.org.uk/vascular/wp-content/ uploads/2012/11/VSGBI-Mortality-Report.pdf

18. EVAR trial participants: Endovascular aneurysm repair and outcome in patients unfit for open repair of abdominal aortic aneurysm (EVAR trial 2): randomised controlled trial. Lancet 2005;365:2187-92.

19. Kothmann E, Batterham AM, Owen SJ, et al. Effect of short-term exercise training on aerobic fitness in patients with abdominal aortic aneurysms: a pilot study. Br J Anaesth 2009;103:505-10.

20. Tew GA, Moss J, Crank $\mathrm{H}$, et al. Endurance exercise training in patients with small abdominal aortic aneurysm: a randomized controlled pilot study. Arch Phys Med Rehabil 2012;93:2148-53.

21. Jack S, West M, Grocott MP. Perioperative exercise training in elderly subjects. Best Pract Res Clin Anaesthesiol 2011; 25:461-72.

22. Wisløff U, Støylen A, Loennechen JP, et al. Superior cardiovascular effect of aerobic interval training versus moderate continuous training in heart failure patients: a randomized study. Circulation 2007;115:3086-94.
23. Guiraud T, Nigam A, Gremeaux V, et al. High-intensity interval training in cardiac rehabilitation. Sports Med 2012 42:587-605.

24. Craig P, Dieppe $P$, Macintyre $S$, et al. Medical Research Council Guidance: developing and evaluating complex interventions: the new Medical Research Council guidance. BMJ 2008;337:a1655.

25. Kothmann E, Danjoux G, Owen SJ, et al. Reliability of the anaerobic threshold in cardiopulmonary exercise testing of patients with abdominal aortic aneurysms. Anaesthesia 2009;64:9-13.

26. EVAR trial participants: Endovascular aneurysm repair versus open repair in patients with abdominal aortic aneurysm (EVAR trial 1): randomised controlled trial. Lancet 2005;365:2179-86.

27. Prinssen M, Buskens E, Blankensteijn JD. DREAM trial participants: quality of life endovascular and open AAA repair. Results of a randomised trial. Eur J Vasc Endovasc Surg 2004;27:121-7.

28. Adamson SJ, Bland JM, Hay EM, et al. Patients' preferences within randomised trials: systematic review and patient level meta-analysis. BMJ 2008;337:a1864.

29. Rognmo $\varnothing$, Moholdt T, Bakken $\mathrm{H}$, et al. Cardiovascular risk of highversus moderate-intensity aerobic exercise in coronary heart disease patients. Circulation 2012;126:1436-40.

30. Moholdt TT, Amundsen BH, Rustad LA, et al. Aerobic interval training versus continuous moderate exercise after coronary artery bypass surgery: a randomized study of cardiovascular effects and quality of life. Am Heart J 2009;158:1031-7.

31. Moholdt T, Aamot IL, Granøien I, et al. Aerobic interval training increases peak oxygen uptake more than usual care exercise training in myocardial infarction patients: a randomized controlled study. Clin Rehabil 2012;26:33-44.

32. Poole DC, Ward SA, Gardner GW, et al. Metabolic and respiratory profile of the upper limit for prolonged exercise in man. Ergonomics 1988;31:1265-79.

33. Borg GA. Psychophysical bases of perceived exertion. Med Sci Sports Exerc 1982;14:377-81.

34. Myers J, Dalman R, Hill B. Exercise, vascular health, and abdominal aortic aneurysms. J Clin Exerc Physiol 2012;1:1-8.

35. Neufer PD. The effect of detraining and reduced training on the physiological adaptations to aerobic exercise training. Sports Med 1989;8:302-20.

36. Stanley DM, Williams SE, Cumming JL. Preliminary validation of a single-item measure of exercise enjoyment: the Exercise Enjoyment Scale. J Sport Exerc Psychol 2009;31:S138-9.

37. Kendzierski D, DeCarlo KJ. Physical Activity Enjoyment Scale: two validation studies. J Sport Exerc Psychol 1991;13:50-64.

38. Myers JN, White JJ, Narasimhan B, et al. Effects of exercise training in patients with abdominal aortic aneurysm: preliminary results from a randomized trial. J Cardiopulm Rehabil Prev 2010; 30:374-83.

39. Moll FL, Powell JT, Fraedrich G, et al. Management of abdominal aortic aneurysms clinical practice guidelines of the European society for vascular surgery. Eur J Vasc Endovasc Surg 2011;41(Suppl 1): S1-58.

40. Hirsch AT, Haskal ZJ, Hertzer NR, et al. ACC/AHA 2005 practice guidelines for the management of patients with peripheral arterial disease (lower extremity, renal, mesenteric and abdominal aortic). Circulation 2006;113:e463-654

41. Best PJ, Tajik AJ, Gibbons RJ, et al. The safety of treadmill exercise stress testing in patients with abdominal aortic aneurysms. Ann Intern Med 1998;129:628-31.

42. Thompson PD, Franklin BA, Balady GJ, et al. American Heart Association Council on Nutrition, Physical Activity, and Metabolism; American Heart Association Council on Clinical Cardiology; American College of Sports Medicine: Exercise and acute cardiovascular events placing the risks into perspective: a scientific statement from the American Heart Association Council on Nutrition Physical Activity, and Metabolism and the Council on Clinical Cardiology. Circulation 2007;115:2358-68.

43. Bennett-Guerrero E, Welsby I, Dunn TJ, et al. The use of a postoperative morbidity survey to evaluate patients with prolonged hospitalization after routine, moderate-risk, elective surgery. Anesth Analg 1999:89:514-19.

44. Grocott MP, Browne JP, Van der Meulen J, et al. The postoperative morbidity survey was validated and used to describe morbidity after major surgery. J Clin Epidemiol 2007;60:919-28.

45. Curtis L. Unit costs of health and social care. PRSSU. University of Kent, 2012.

46. Department of Health. NHS reference costs: financial year 2011 to 2012. www.gov.uk/government/uploads/system/uploads/ attachment_data/file/213060/2011-12-reference-costs-publication.pdf (accessed Nov 2013) 